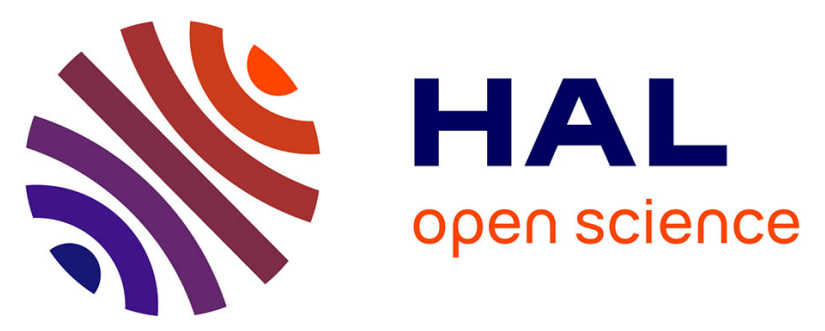

\title{
Mass sensor using mode localization in two weakly coupled MEMS cantilevers with different lengths: Design and experimental model validation
}

Toky Harrison Rabenimanana, Vincent Walter, Najib Kacem, Patrice Le Moal, Gilles Bourbon, Joseph Lardies

\section{To cite this version:}

Toky Harrison Rabenimanana, Vincent Walter, Najib Kacem, Patrice Le Moal, Gilles Bourbon, et al.. Mass sensor using mode localization in two weakly coupled MEMS cantilevers with different lengths: Design and experimental model validation. Sensors and Actuators A: Physical , 2019, 295, pp.643 652. hal-02300032

\section{HAL Id: hal-02300032 https://hal.science/hal-02300032}

Submitted on 29 Sep 2019

HAL is a multi-disciplinary open access archive for the deposit and dissemination of scientific research documents, whether they are published or not. The documents may come from teaching and research institutions in France or abroad, or from public or private research centers.
L'archive ouverte pluridisciplinaire $\mathbf{H A L}$, est destinée au dépôt et à la diffusion de documents scientifiques de niveau recherche, publiés ou non, émanant des établissements d'enseignement et de recherche français ou étrangers, des laboratoires publics ou privés. 


\title{
Mass sensor using mode localization in two weakly coupled MEMS cantilevers with different lengths: design and experimental model validation
}

Toky Rabenimanana*, Vincent Walter, Najib Kacem, Patrice Le Moal, Gilles Bourbon, Joseph Lardiès

Univ. Bourgogne Franche-Comté, FEMTO-ST Institute, CNRS/UFC/ENSMM/UTBM, Department of Applied Mechanics, France

\begin{abstract}
This paper presents a sensor using the mode localization phenomenon to detect a mass perturbation. It is composed of two cantilevers with different lengths and connected by a coupling beam. The short cantilever is electrostatically actuated and by changing the applied DC voltage, we can reduce its stiffness and reach the veering point, which corresponds to a balanced system. This principle allows us to overcome the manufacturing defect which perturbs the initial system. An analytical model using the Euler-Bernoulli beam theory is developed for the design. The equation of the continuous system is discretized with the Galerkin method and simulations are performed. The designed device composed of polysilicon coupled microbeams is then fabricated with the Multi-User MEMS Processes and an experimental investigation is carried out. Three devices with different coupling are considered with a length ratio of 0.98 . This ratio is suitable to reach the veering point by using a DC balancing voltage around the half of the pull-in voltage. The comparison between theoretical and experimental results shows a good agreement for each device.
\end{abstract}

Keywords: mode localization, manufacturing defects, veering point, electrostatic actuation, mechanical coupling

\section{Introduction}

Among the many and diversified applications of Microelectromechanical systems (MEMS), mass detection is a promising one for detecting and measuring the presence of biological species like viruses or bacteria, whether in liquid or gaseous media. Most of these sensors use the change in the resonant frequency $[1,2,3,4]$ as output signal and it is possible to improve their sensitivity by using nanoelectromechanical systems (NEMS) [5] which have a high resonant frequency. But nanometer scale devices present the disadvantage of being more susceptible to noises [6] and their linear dynamic range is limited without nonlinearities cancellation $[7,8,9]$. Other alternatives for mass sensors have been presented in the literature. Zhang et al. [10] used a mass sensor based on parametric resonance. Kumar et al. [11]

\footnotetext{
*Corresponding author

Email address: toky.rabenimanana@femto-st.fr (Toky Rabenimanana)
} 
studied bifurcation-based mass sensors which measure the sudden change in the amplitude response of nonlinear resonators. Kacem et al. [12] proposed to use simultaneous resonance to overcome the limitations of nanomechanical resonators. However, all of these devices is composed of a single resonator and the ambient condition can change their output signal [13].

Recently, a new generation of mass sensors composed of coupled resonators has emerged. This kind of sensor uses the phenomenon of mode localization $[14,15]$ and measures the change in the vibration mode of coupled resonators to detect a small perturbation. The normalized sensitivity of this sensor depends on the ratio between the stiffness of the resonator and of the coupling. When the coupling is very weak, this normalized sensitivity can be two to three orders of magnitude higher than the sensitivity of a device using frequency variations [13]. The use of multiple resonators has also the advantage of improving common mode rejection capabilities [16]. Therefore, mass sensors using weakly coupled resonators have been considered in recent publications. Spletzer et al. [17] presented a mode localized mass sensor using two cantilevers coupled by an overhang, and a mass of $154 \mathrm{pg}$ was placed on their device to highlight the mode localization. Thiruvenkatanathan et al. [18] used a mass sensor with two electrically coupled resonators. Chopard et al. [19] studied two coupled cantilever mass sensor with piezoelectric excitation. Other authors considered devices using more resonators, like Wang et al. [20], which used a mass sensor based on three degrees of freedom coupled resonator and operating in air, or Spletzer et al. [21], which considered a device with fifteen weakly coupled cantilevers. This kind of device also concerns stiffness sensors [22, 23] and acceleration sensors [24].

In the case of two identical coupled resonators, the vibration modes corresponding to the system without any perturbation are the symmetric mode noted $(1 ; 1)$ and the antisymmetric mode noted $(1 ;-1)$. In these two modes, the vibrations are respectively in-phase and out-of-phase and we have identical amplitudes on both resonators. For the detection, the device needs to be at the veering point corresponding to these two modes. They appear when we have exactly two identical resonators. But when the size of the device decreases, the manufacturing defects become non-negligible. So, the device is initially unbalanced and localized vibrations take place without any additional perturbation. In order to overcome these defects, we propose to use a device composed of two coupled cantilevers with different lengths. With this configuration, the short cantilever has a smaller mass and a higher stiffness. As the short cantilever is electrostatically actuated with a combined AC-DC voltage, we use the softening effect of the electrostatic force to reduce its stiffness. By applying the appropriate DC voltage, we can balance the device and reach the veering point. Tuning the DC voltage to counterbalance defects can be realized with two resonators with the same dimensions, but using initial asymmetric device allows us to know which one is the stiffest and needs electrostatic softening. This principle was already investigated in [25] and an analytical model using the Euler-Bernoulli beam theory was briefly exposed in [26]. In this paper, we will detail this model in order to design the device. Concerning the mechanical coupling, most of sensors use an overhang to connect the two cantilevers but its design is not accurately modeled. As the coupling is a parameter which defines the sensitivity of the device, it needs to be correctly designed. Therefore, we propose to use a coupling beam to connect the two cantilevers, and it is taken into account in the model. The sensor is then fabricated with the Multi-User MEMS Processes for an experimental investigation. Three 
devices with different coupling are tested to validate the proposed model.

\section{Device description and model}

The device used in this study (Fig. 1a) is composed of two micro cantilevers with lengths $L_{1}$ and $L_{2}\left(L_{1}<L_{2}\right)$, a width $b$ and a thickness $h$. The coupling plays a major role in defining the device sensitivity, that's why its design and dimensions have to be controllable. Most of sensors use an overhang to connect both cantilevers. Such a coupling is difficult to be accurately modeled and as a consequence practically uncontrollable. Therefore, a coupling beam with a length $L_{c}$, a width $b_{c}$, a thickness $h$ is used to connect the two cantilevers at a distance $\tilde{x}_{c}$ from the fixed end. For the electrostatic actuation, we apply a combined AC-DC voltage to the electrode located at a distance $g$ from the short cantilever.

This device is modeled with two Euler Bernoulli beams connected by a rotational spring $\tilde{k}_{r}$ (Fig. 1b). This represents the torsional stiffness [27] of the coupling beam and it is expressed as

$$
\tilde{k}_{r}=\frac{G \beta b_{c} h^{3}}{L_{c}}
$$

where $G$ is the shear modulus of the constitutive material and $\beta$ is a coefficient depending on the ratio $b_{c} / h$. A model of mechanically coupled microbeams has already been presented in [28] and the coupling beam was also modeled with an Euler Bernoulli beam subjected to a bending stress. But in our case, the coupling beam is near the fixed end and the dominant stress is the torsion. So, the mass and the bending stiffness of the coupling beam are neglected in our model. As the surface micromachining process does not allow ideal clamping conditions to be achieved, a torsional stiffness $\tilde{k}_{e}$ is introduced in the model at the supposed fixed ends. Normally, a complete modelization of the real boundary condition should include a translational spring $[29,30]$ but we assume that the small deflection at the fixed end can be neglected. On the long cantilever, we also add a discrete point mass located at a distance $\tilde{x}_{m}$ from the fixed end in order to consider the mass perturbation.

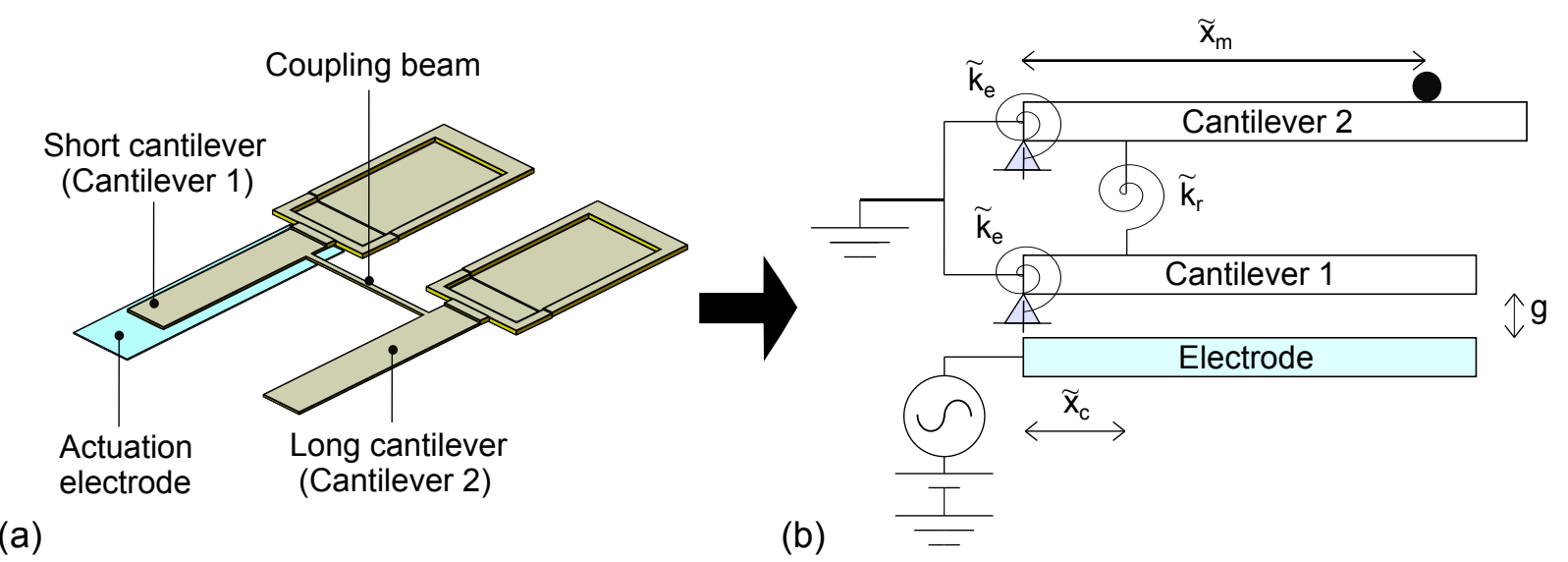

Figure 1: The device (a) and the model (b).

According to the equation of beams carrying lumped element [31, 32, 33], the equations 
governing the bending vibration of the model shown in Fig. 1b is given by

$$
\begin{gathered}
E I \tilde{w}_{1}^{\prime \prime \prime \prime}+\rho b h \ddot{\tilde{w}}_{1}+\tilde{c}_{1} \dot{\tilde{w}}_{1}-\tilde{k}_{r}\left(\tilde{w}_{1}^{\prime}\left(\tilde{x}_{c}\right)-\tilde{w}_{2}^{\prime}\left(\tilde{x}_{c}\right)\right) \delta^{\prime}\left(\tilde{x}-\tilde{x}_{c}\right)=\frac{1}{2} \varepsilon_{0} b C_{n} \frac{\left(V_{D C}+v_{A C} \cos (\tilde{\Omega} \tilde{t})\right)^{2}}{\left(g-\tilde{w}_{1}\right)^{2}} \\
E I \tilde{w}_{2}^{\prime \prime \prime \prime}+\left(\rho b h+\tilde{m} \delta\left(\tilde{x}-\tilde{x}_{m}\right)\right) \ddot{\tilde{w}}_{2}+\tilde{c}_{2} \dot{\tilde{w}}_{2}-\tilde{k}_{r}\left(\tilde{w}_{2}^{\prime}\left(\tilde{x}_{c}\right)-\tilde{w}_{1}^{\prime}\left(\tilde{x}_{c}\right)\right) \delta^{\prime}\left(\tilde{x}-\tilde{x}_{c}\right)=0
\end{gathered}
$$

where primes and dots denote respectively the partial differentiation with respect to the position along the microbeam $\tilde{x}$ and to the time $\tilde{t}, E$ is the Young's modulus of the constitutive material, $I$ is the moment of inertia of the cross section, $\tilde{w}_{1}$ and $\tilde{w}_{2}$ are respectively the deflection of the first (shortest) and the second (longest) cantilever, $\tilde{c}_{1}$ and $\tilde{c}_{2}$ are the damping coefficient, $\delta$ is the Dirac delta function, $\rho$ is the material density, $\varepsilon_{0}$ is the dielectric constant of the medium between the cantilever 1 and the actuation electrode, $C_{n}$ is a coefficient which takes into account the fringing field effects [34], $V_{D C}$ and $v_{A C}$ are respectively the DC and AC actuation voltage, $\tilde{\Omega}$ is the excitation frequency and $g$ is the capacitor gap. The damping coefficient of each cantilever is given by [9]

$$
\tilde{c}_{i}=\frac{2 \pi f_{i} \rho b h}{Q} \quad i=1,2
$$

where $f_{i}$ is the resonant frequency of the cantilever $i$ and $\mathrm{Q}$ is the quality factor. For convenience, we introduce the non-dimensional variables and parameters below

$$
\begin{gathered}
x=\frac{\tilde{x}}{L_{1}} ; x_{c}=\frac{\tilde{x}_{c}}{L_{1}} ; x_{m}=\frac{\tilde{x}_{m}}{L_{1}} ; m=\frac{\tilde{m}}{\rho b h L_{1}} ; w_{i}=\frac{\tilde{w}_{i}}{g} ; \tau=L_{1}^{2} \sqrt{\frac{\rho b h}{E I}} \\
t=\frac{\tilde{t}}{\tau} ; \Omega=\tilde{\Omega} \tau ; c_{i}=\frac{L_{1}^{4} \tilde{c}_{i}}{E I \tau} ; k_{r}=\frac{\tilde{k}_{r} L_{1}}{E I} ; \delta_{0}=\frac{1}{2} \varepsilon_{0} b C_{n} \frac{L_{1}{ }^{4}}{g^{3} E I}
\end{gathered}
$$

and the electrostatic force is expanded into a third order Taylor series. As the AC voltage is also negligible in comparison with the DC voltage, we can drop the terms with $v_{A C}{ }^{2}$ when developing the electrostatic force. After these considerations, we obtain the following non-dimensional equations

$$
\begin{array}{r}
w_{1}^{\prime \prime \prime \prime}+\ddot{w}_{1}+c_{1} \dot{w}_{1}-k_{r}\left(w_{1}^{\prime}\left(x_{c}\right)-w_{2}^{\prime}\left(x_{c}\right)\right) \delta^{\prime}\left(x-x_{c}\right)=\delta_{0}\left(V_{D C}^{2}+2 V_{D C} v_{A C} \cos (\Omega t)\right) \\
*\left(1+2 w_{1}+3 w_{1}^{2}+4 w_{1}^{3}\right) \\
w_{2}^{\prime \prime \prime \prime}+\left(1+m \delta\left(x-x_{m}\right)\right) \ddot{w}_{2}+c_{2} \dot{w}_{2}-k_{r}\left(w_{2}^{\prime}\left(x_{c}\right)-w_{1}^{\prime}\left(x_{c}\right)\right) \delta^{\prime}\left(x-x_{c}\right)=0
\end{array}
$$

where primes and dots denote respectively the partial differentiation with respect to the new non-dimensional variables $x$ and $t$. When the electrostatic actuation is composed of a DC and $\mathrm{AC}$ voltage, the microbeam is deflected by the $\mathrm{DC}$ component and vibrates by the $\mathrm{AC}$ component. Therefore, the equilibrium position of the microbeam is shifted and it vibrates around a new static deflection which depends on the DC voltage. By using one mode Galerkin discretization, the total deflection of each cantilever can be expressed by [35]

$$
w_{1}(x, t)=\left(a_{1 s}+a_{1}(t)\right) * \phi_{1}(x) ; w_{2}(x, t)=\left(a_{2 s}+a_{2}(t)\right) * \phi_{2}(x)
$$


where $a_{1 s}$ and $a_{2 s}$ are generalized coordinates of the static deflection, $a_{1}(t)$ and $a_{2}(t)$ are the time varying generalized coordinates and $\phi_{1}(x)$ and $\phi_{2}(x)$ are the mode shapes. As the coupling is weak, we assume that the mode shape of each cantilever is the same as the mode shape of single cantilever without the coupling, so $\phi_{1}(x)$ and $\phi_{2}(x)$ are given by

$$
\begin{aligned}
& \phi_{i}(x)=K_{i 1}\left[K_{i 2} \sin \left(\lambda_{i} x \frac{L_{1}}{L_{i}}\right)-K_{i 3} \sinh \left(\lambda_{i} x \frac{L_{1}}{L_{i}}\right)\right. \\
& \left.+\frac{\sin \lambda_{i}+\sinh \lambda_{i}}{\cos \lambda_{i}+\cosh \lambda_{i}}\left(\cosh \left(\lambda_{i} x \frac{L_{1}}{L_{i}}\right)-\cos \left(\lambda_{i} x \frac{L_{1}}{L_{i}}\right)\right)\right]
\end{aligned}
$$

where $K_{i 1}$ are chosen in order to have

$$
\int_{0}^{\frac{L_{i}}{L_{1}}} \phi_{i}^{2}(x) d x=1
$$

$K_{i 2}$ and $K_{i 3}$ are expressed as

$$
K_{i 2}=\frac{\cos \lambda_{i}+\cosh \lambda_{i}+\frac{2 E I \lambda_{i}}{\tilde{k}_{e} L_{i}} \sinh \lambda_{i}}{\cos \lambda_{i}+\cosh \lambda_{i}} ; K_{i 3}=\frac{\cos \lambda_{i}+\cosh \lambda_{i}-\frac{2 E I \lambda_{i}}{\tilde{k}_{e} L_{i}} \sin \lambda_{i}}{\cos \lambda_{i}+\cosh \lambda_{i}}
$$

and $\lambda_{i}$ is the first solution of the following equation

$$
\frac{\tilde{k}_{e} L_{i}}{E I \lambda_{i}}\left(\cos \lambda_{i} \cosh \lambda_{i}+1\right)+\cos \lambda_{i} \sinh \lambda_{i}-\sin \lambda_{i} \cosh \lambda_{i}=0
$$

Then, we multiply respectively Eq. (6) and Eq. (7) by $\phi_{1}(x)$ and $\phi_{2}(x)$ and integrate them respectively from $x=0$ to $x=1$ and from $x=0$ to $x=L_{2} / L_{1}$. When the value of the torsional stiffness $\tilde{k}_{e}$ at the fixed end is sufficiently high to ensure a good clamping condition, we obtain the following parameters which remain almost unchanged

$$
\int_{0}^{1} \phi_{1}(x) d x \approx 0.784 ; 3 \int_{0}^{1} \phi_{1}^{3}(x) d x \approx 4.43 ; 4 \int_{0}^{1} \phi_{1}^{4}(x) d x \approx 9.39
$$

By considering the following properties of the Dirac delta function

$$
\int_{0}^{\frac{L_{2}}{L_{1}}} \delta\left(x-x_{m}\right) \phi_{2}^{2}(x) d x=\phi_{2}^{2}\left(x_{m}\right) ; \int_{0}^{\frac{L_{i}}{L_{1}}} \delta^{\prime}\left(x-x_{c}\right) \phi_{i}(x) d x=-\phi_{i}{ }^{\prime}\left(x_{c}\right)
$$

we deduce from Eqs. $(6,7,8,9)$ and Eq. (13)

$$
\begin{aligned}
& \left(a_{1 s}+a_{1}\right) \lambda_{1}{ }^{4}+\ddot{a}_{1}+c_{1} \dot{a}_{1}+k_{r}\left(\left(a_{1 s}+a_{1}\right) \phi_{1}^{\prime 2}\left(x_{c}\right)-\left(a_{2 s}+a_{2}\right) \phi_{1}^{\prime}\left(x_{c}\right) \phi_{2}^{\prime}\left(x_{c}\right)\right) \\
& =\delta_{0}\left(V_{D C}{ }^{2}+2 V_{D C} v_{A C} \cos (\Omega t)\right)\left(0.784+2\left(a_{1 s}+a_{1}\right)+4.43\left(a_{1 s}+a_{1}\right)^{2}+9.39\left(a_{1 s}+a_{1}\right)^{3}\right) \\
& \left(a_{2 s}+a_{2}\right) \lambda_{2}{ }^{4} \frac{L_{1}{ }^{4}}{L_{2}{ }^{4}}+\left(1+m \phi_{2}^{2}\left(x_{m}\right)\right) \ddot{a}_{2}+c_{2} \dot{a}_{2} \\
& +k_{r}\left(\left(a_{2 s}+a_{2}\right) \phi_{2}^{\prime 2}\left(x_{c}\right)-\left(a_{1 s}+a_{1}\right) \phi_{1}^{\prime}\left(x_{c}\right) \phi_{2}^{\prime}\left(x_{c}\right)\right)=0
\end{aligned}
$$


Firstly, the static deflection is calculated by dropping all time varying terms [35] in Eq. (15) and Eq. (16), and we obtain

$$
\begin{aligned}
& a_{1 s} \lambda_{1}{ }^{4}+k_{r}\left(a_{1 s} \phi_{1}^{\prime 2}\left(x_{c}\right)-a_{2 s} \phi_{1}^{\prime}\left(x_{c}\right) \phi_{2}^{\prime}\left(x_{c}\right)\right)=\delta_{0} V_{D C}{ }^{2} \\
& *\left(0.784+2 a_{1 s}+4.43 a_{1 s}{ }^{2}+9.39 a_{1 s}{ }^{3}\right) \\
& a_{2 s} \lambda_{2}{ }^{4} \frac{L_{1}{ }^{4}}{L_{2}{ }^{4}}+k_{r}\left(a_{2 s} \phi_{2}^{\prime 2}\left(x_{c}\right)-a_{1 s} \phi_{1}^{\prime}\left(x_{c}\right) \phi_{2}^{\prime}\left(x_{c}\right)\right)=0
\end{aligned}
$$

Then, Eq. (15) and Eq. (16) are developed and we can eliminate some terms by considering Eq. (17) and Eq. (18). As we use small vibration amplitudes which are under $10 \%$ of the capacitor gap, we assume that the vibration is still linear. By keeping only the harmonic load and dropping all nonlinear terms, we finally obtain the matrix equation below

$$
[M]\left[\begin{array}{l}
\ddot{a}_{1} \\
\ddot{a}_{2}
\end{array}\right]+[C]\left[\begin{array}{c}
\dot{a}_{1} \\
\dot{a}_{2}
\end{array}\right]+[K]\left[\begin{array}{l}
a_{1} \\
a_{2}
\end{array}\right]=[F] * \cos (\Omega t)
$$

where

$$
\begin{aligned}
& {[M]=\left[\begin{array}{cc}
1 & 0 \\
0 & 1+m \phi_{2}^{2}\left(x_{m}\right)
\end{array}\right] ;[C]=\left[\begin{array}{cc}
c_{1} & 0 \\
0 & c_{2}
\end{array}\right]} \\
& {[K]=\left[\begin{array}{cc}
\lambda_{1}^{4}-\delta_{0} V_{D C}^{2}\left(2+8.86 a_{1 s}+28.17 a_{1 s}^{2}\right)+k_{r} \phi_{1}^{\prime 2}\left(x_{c}\right) & -k_{r} \phi_{1}^{\prime}\left(x_{c}\right) \phi_{2}^{\prime}\left(x_{c}\right) \\
-k_{r} \phi_{1}^{\prime}\left(x_{c}\right) \phi_{2}^{\prime}\left(x_{c}\right) & \lambda_{2}{ }^{4} \frac{L_{1}{ }^{4}}{L_{2}{ }^{4}}+k_{r} \phi_{2}^{\prime 2}\left(x_{c}\right)
\end{array}\right] ;} \\
& {[F]=\left[\begin{array}{c}
\left(1.56+4 a_{1 s}+8.86 a_{1 s}^{2}+18.78 a_{1 s}{ }^{3}\right) \delta_{0} v_{A C} V_{D C} \\
0
\end{array}\right]}
\end{aligned}
$$

Eq. (19) is similar to the equation of two degrees of freedom system and solving this equation gives us the value of $A_{1}$ and $A_{2}$ which are respectively the amplitude of $a_{1}$ and $a_{2}$. Finally, the frequency $(f)$ and the vibration amplitude at the end of each cantilever $\left(W_{1}\right.$ and $\left.W_{2}\right)$ are given by

$$
f=\frac{\Omega}{2 \pi \tau} ; W_{1}=A_{1} * \phi_{1}(1) * g ; W_{2}=A_{2} * \phi_{2}\left(\frac{L_{2}}{L_{1}}\right) * g
$$

\section{Design and fabrication of the device}

To design the device, we use the proposed model with the following dimensions: $b=$ $20 \mu m ; h=1.30 \mu m ; g=1.17 \mu m ; L_{c}=65 \mu m ; b_{c}=3 \mu m$ and $\tilde{x}_{c}=10.5 \mu m$. With these dimensions, the parameter $\beta$ in Eq. (1) is equal to 0.242 and the coefficient for the fringing field effects is $C_{n}=1.13$. For the other parameters, we take the values given in Table 1 . In this table, the Young's modulus and the shear modulus correspond to the material properties of polysilicon, and $Q$ is based on a quality factor of a single cantilever of a previous design. The length of the long cantilever has been fixed at $100 \mu \mathrm{m}$. The length of the short cantilever has been determined so that the DC balancing voltage is less than half of the static pull-in voltage [36]. To do so, a series of simulations has been launched for a length of the short cantilever in the range $96 \mu \mathrm{m}$ to $100 \mu \mathrm{m}$. For each value, the AC voltage is $v_{A C}=10 \mathrm{mV}$ 
and the DC balancing voltage corresponding to the veering point has been determined. The DC balancing voltage is reached when the system exhibits the symmetric and antisymmetric modes. As there is no mass perturbation, the value of $m$ in the matrix $[M]$ in Eq. (20) is equal to zero. The Fig. 2 shows the results in terms of pull-in and balancing voltage for different

Table 1: Parameters for the simulation

\begin{tabular}{cc}
\hline Parameters & Value \\
\hline$E[G P a]$ & 169 \\
$G[G P a]$ & 69.3 \\
$\rho\left[\mathrm{kgm}^{-3}\right]$ & 2320 \\
$\varepsilon_{0}\left[\mathrm{~m}^{-3} \mathrm{~kg}^{-1} \mathrm{~s}^{4} \mathrm{~A}^{2}\right]$ & $8.8510^{-12}$ \\
$\tilde{k}_{e}[\mathrm{Nm} / \mathrm{rad}]$ & $1.5010^{-7}$ \\
$Q$ & 450 \\
\hline
\end{tabular}

lengths of the short cantilever.

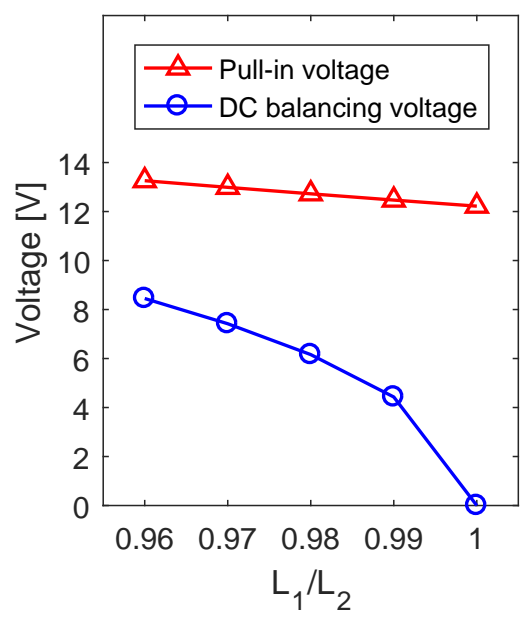

Figure 2: DC balancing voltage and pull-in voltage as a function of the length ratio between the two cantilevers.

In Fig. 2, the DC voltage that we have to use is close to the pull-in voltage when the length difference between the two cantilevers increases. By choosing a length ratio of 0.98 , we get a DC balancing voltage around the half of this pull-in voltage, so the length of the short cantilever is fixed at $98 \mu \mathrm{m}$.

After choosing the lengths of the cantilevers, the designed device was fabricated by using the Multi-User MEMS Processes (MUMPsß). As shown in Fig. 3, the microbeams are composed of a polysilicon layer Poly2 $(1.30 \mu \mathrm{m}$ thick) renforced by a second polysilicon layer Poly1 $(1.85 \mu \mathrm{m}$ thick) at the fixed end. The measured gap between the cantilevers and the bottom electrode (Poly0) is around $1.17 \mu \mathrm{m}$. On the chip containing the cantilevers, three different devices were considered (Fig. 4) with coupling beam locations at $\tilde{x}_{c}=5.5 \mu \mathrm{m}$, $\tilde{x}_{c}=10.5 \mu \mathrm{m}$ and $\tilde{x}_{c}=15.5 \mu \mathrm{m}$ from the fixed end. Changing the value of $\tilde{x}_{c}$ allows us to change the coupling parameter and it is weaker when the coupling beam is near the fixed 
end. There are electrodes under both cantilevers, but only the one under the short cantilever is actuated.
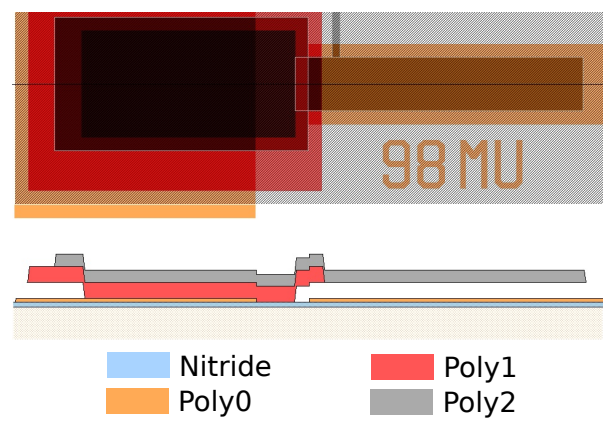

Figure 3: Top view and cross section of the short cantilever $\left(L_{1}=98 \mu \mathrm{m}\right)$.
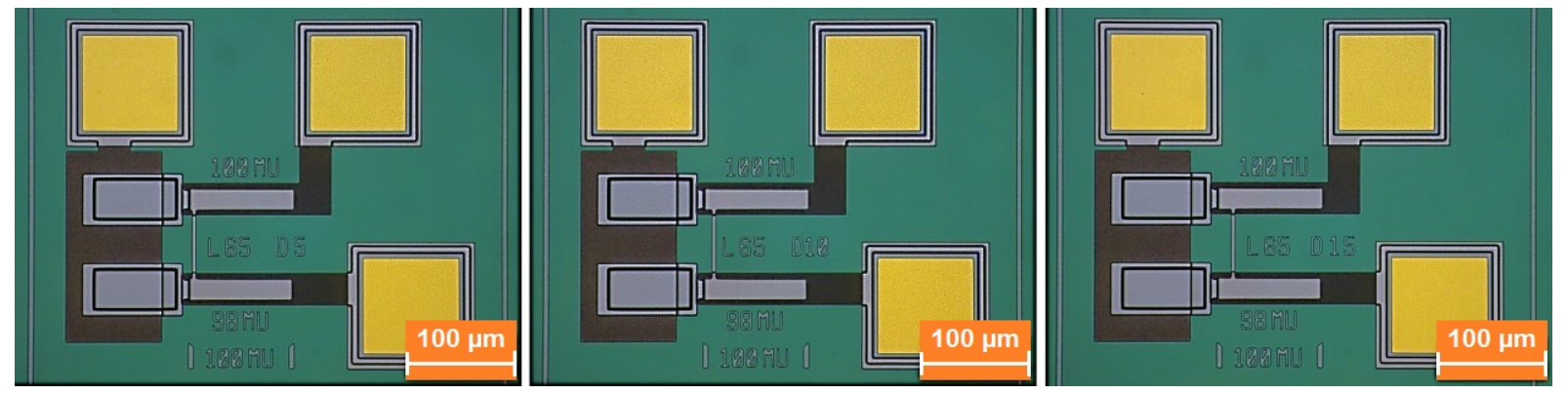

Figure 4: Optical microscope images of the three fabricated devices (from left to right, $\tilde{x}_{c}=5.5 \mu m, \tilde{x}_{c}=$ $10.5 \mu m$ and $\tilde{x}_{c}=15.5 \mu m$ )

\section{Experimental setup and model validation}

To realize the experimental setup, the chip containing the coupled MEMS cantilevers is placed in a vacuum chamber (Fig. 5a) to decrease the pressure at 0.50 mbar. The vibration is then generated by using probes connected to a generator. To determine the vibration amplitude, the vibration velocity at the end of each cantilever is measured by a single point laser Doppler vibrometer Polytec OFV-5000. We deduce then the vibration amplitude from the velocity amplitude. Here, the results of interest are the frequency responses of each cantilever so we use a Picoscope $\cap 5444 \mathrm{~B}$ to automatically collect the data. As detailed in Fig. 5b, this PC based oscilloscope is piloted with a MATLAB program to produce the AC actuation and to obtain the signal coming from the laser Doppler vibrometer. The vibration amplitudes can also be measured with other methods such as electrical readout. In this case, we detect the motional current due to the capacitance variation and it can be amplified with CMOS readout circuit [9].

For the theoretical results, we use the developed model and perform the simulation with the given dimensions and parameters. As there is no mass perturbation, the parameter $m$ in Eq. (20) is still equal to zero. However, there are some parameters which are identified experimentally. The first parameter is the quality factor Q. For cantilevers vibrating in 


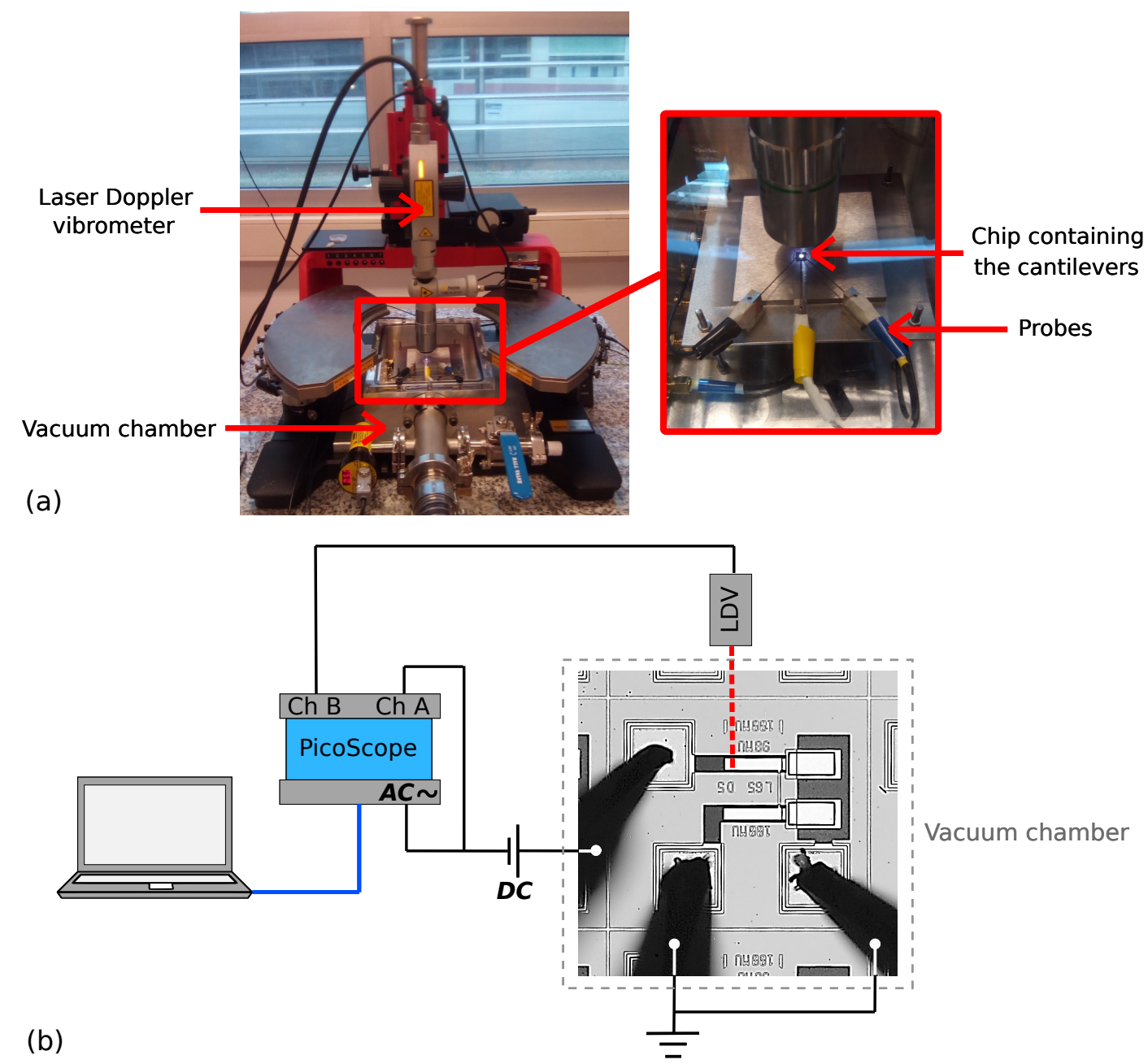

Figure 5: The laser Doppler vibrometer used for experiments (a) and schematic of the experimental setup (b).

air, the quality factor depends on extrinsic loss (viscous losses and acoustic radiation) and intrinsic loss (support loss, thermoelastic damping, volume loss and surface loss) [37]. For cantilevers vibrating at moderately low pressure, the viscous damping with rarefied gas effect is shown to be dominant [38]. In addition to it, attachment loss [39] can also be among the main damping source. But all of these losses mechanisms are complex and it does not allow us to estimate the quality factor with a high accuracy. So, it is determined directly from experimental results by using the half-power bandwidth method on a single cantilever on the same chip and with the same dimensions. The second parameter is the torsional spring which modelizes the actual clamping condition. At the veering point, the coupling beam does not interfere in the vibration when the two cantilevers vibrate in phase. Therefore, the frequency of the symmetric mode is equal to the natural frequency of each resonator, i.e. the natural frequency of the long cantilever or the natural frequency of the short cantilever under the softening effect of the DC voltage. By analyzing the free vibration of a single cantilever with a length $L_{2}$ and a torsional spring $\tilde{k}_{e}$ at its end, we can deduce from the experimental 
frequency $f_{1 \text { exp }}$ of the device in the symmetric mode that

$$
\tilde{k}_{e}=\frac{E I \frac{\lambda_{2}}{L_{2}}\left(-\cos \lambda_{2} \sinh \lambda_{2}+\sin \lambda_{2} \cosh \lambda_{2}\right)}{\cos \lambda_{2} \cosh \lambda_{2}+1} \quad \text { where } \lambda_{2}=L_{2} \sqrt{2 \pi f_{1 \exp } \sqrt{\frac{\rho b h}{E I}}}
$$

And the last parameter is the capacitor gap $g$. As the measured value is not very accurate, we chose to deduce it from the experimental DC balancing voltage.

\section{Results}

\subsection{Frequency loci veering and amplitude ratio}

First, the frequencies and the amplitude ratios of the two cantilevers $\left(W_{1} / W_{2}\right)$ in each mode (the in-phase mode and the out-of-phase mode) are plotted as a function of the applied $\mathrm{DC}$ voltage. Then, the simulation is performed with the parameters given in Table 2 which have been identified at the veering point. For simplification, we assume that the two cantilevers have the same damping coefficient $\left(\tilde{c}_{1}=\tilde{c}_{2}\right)$ and we determine it with Eq. (4). The results obtained from the simulation and from the experimentation are compared as shown in Fig. 6.

Table 2: Identified parameters for the model.

\begin{tabular}{cccc}
\hline Parameters & Device 1 & Device 2 & Device 3 \\
\hline$Q$ & 450 & 450 & 450 \\
$\tilde{k}_{e}[N \mathrm{~m} / \mathrm{rad}]$ & $1.5410^{-7}$ & $1.5010^{-7}$ & $1.4210^{-7}$ \\
$g[\mu \mathrm{m}]$ & 1.16 & 1.13 & 1.16 \\
\hline
\end{tabular}

At the veering point, we reach the balanced state. This corresponds to the case where the frequencies of each mode (Fig. 6a) are the closest to each other and the amplitude ratio (Fig. 6b) is equal to one. At this state, the mode shapes of the coupled microbeams are similar to those of a device with two cantilevers exactly identical. They correspond to the symmetric and anti-symmetric modes, as shown in Fig. 7. At this balanced state, the experimental DC balancing voltage for device 1 , device 2 and device 3 are respectively $V_{D C 1}=6.10 \mathrm{~V}$, $V_{D C 2}=5.85 \mathrm{~V}$ and $V_{D C 3}=6.10 \mathrm{~V}$. These three voltages are different because the identified gap (in Table 2) to have the same results in the model are different. However, they are all close to the value measured with an interferometer (around $1.17 \mu \mathrm{m}$ ). Concerning the in-phase mode at the veering point (symmetric mode), we also notice that each device has experimentally a different frequency. We have $f_{1}=166.3 \mathrm{kHz}$ for device $1, f_{1}=166.0 \mathrm{kHz}$ for device 2 and $f_{1}=165.3 \mathrm{kHz}$ for device 3 . Theoretically, only the frequency of the antisymmetric mode depends on the coupling parameter but the frequency of the symmetric mode remains unchanged. However, the clamping condition is not exactly identical for each microbeam so their natural frequencies are different. As we can see in Table 2, the identified torsional spring which modelizes the clamping condition is different for each device. But these differences are also due to other small imperfections such as the non-uniformity of the thicknesses or planar dimensions. 

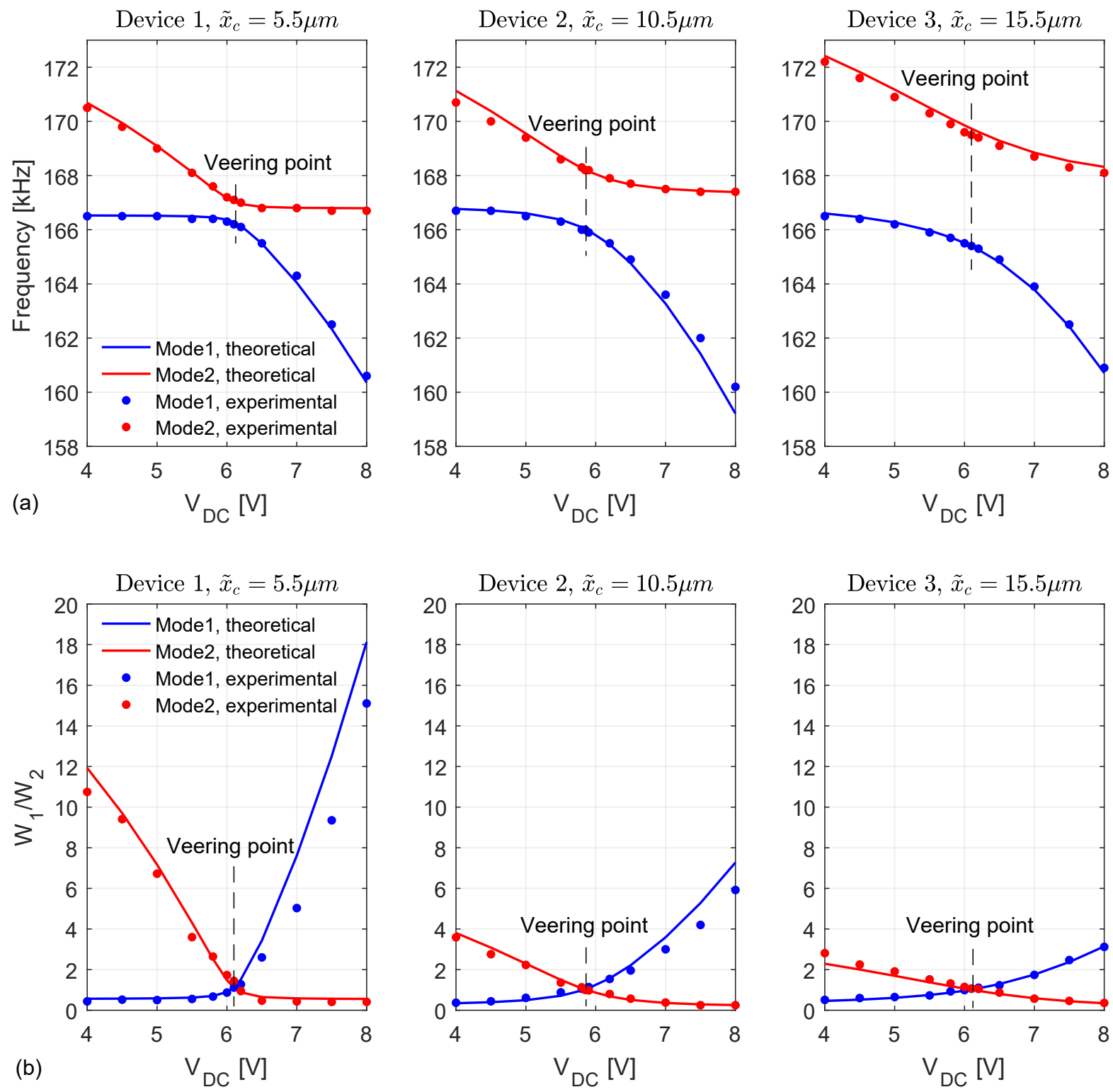

Figure 6: Frequencies of each mode (a) and amplitude ratio of the two cantilevers in each mode (b) as a function of the DC voltage.

For the pull-in instability, the DC balancing voltage that we use are all low compared to the static pull-in voltage which is around $12 \mathrm{~V}$. It means that the length difference between the two designed cantilevers is suitable for the device.

By varying the applied DC voltage, we obtain the frequency loci veering (Fig. 6a) [40] and the model fits the experimental results. On each mode, there is a little difference between the frequencies of each microbeams and the frequency of the short cantilever was considered for this figure. When we are not at the veering point, the amplitude ratio in Fig. 6b shows that the vibration to either side of the balancing state is localized. As reported in [41], the vibration of the first mode is localized in the softest microbeam and that of the second mode is localized in the stiffest microbeam. With the same applied DC voltage, the vibration is 


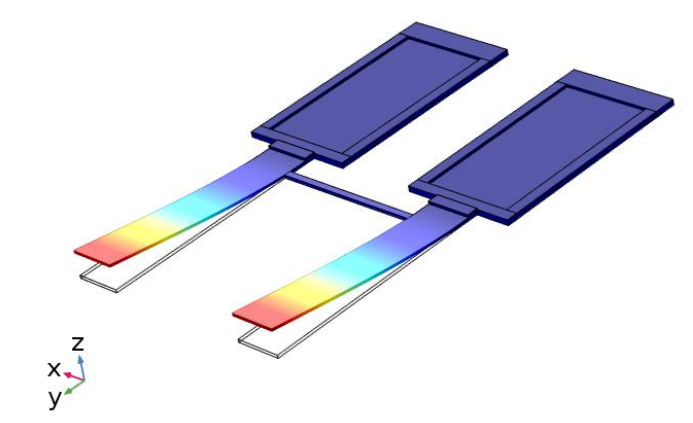

(a)

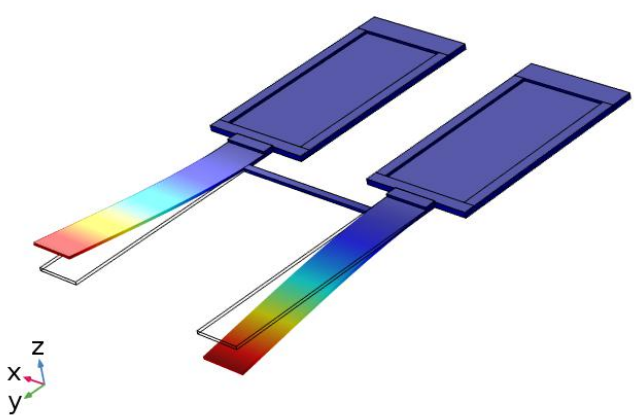

(b)

Figure 7: Mode shapes of the device at the veering point: the symmetric mode (a) and the anti-symmetric mode (b)

more localized in device 1 . Indeed, a DC voltage not corresponding to the balanced state constitutes a stiffness perturbation and the device with the weakest coupling is the most sensitive. The theoretical results of the amplitude ratio show a curve with the same shape, but the difference with the experimental results is more important when the DC voltage is too low or too high. In device 1 , these cases correspond to the mode $(1 ; 0)$ or the mode $(0 ; 1)$. In these modes, the vibration is localized in one of the two cantilevers (an amplitude ratio around 15 for the in-phase mode when $V_{D C}=8 \mathrm{~V}$ ) so the vibration amplitude of the other one becomes too small to be accurately measured.

\subsection{Frequency responses at the veering point}

We consider now the device 2 and its frequency responses at the veering point are shown in Fig. 8. The AC voltage for the excitation is $v_{A C}=10 \mathrm{mV}$ and the DC voltage is $V_{D C}=5.85 \mathrm{~V}$, as previously mentioned. The amplitude response shows that we have the same amplitude on both cantilevers at the resonance frequency so we get the symmetric and the anti-symmetric modes. The comparison also shows that there is a good agreement between both results obtained from the model and from experimental measurement. As the difference between the frequencies of the two modes depends on the coupling parameter, this result implies that the modeling of the coupling beam is validated. It can also be verified in the two other devices by comparing the frequencies at the veering point (Fig. 6a). Concerning the phase response, we note in Fig. 8c that the phase shift between the vibrations of the two cantilevers and the excitation is equal to $-90^{\circ}$ for the symmetric mode. This same phase shift means that the two cantilevers vibrate in-phase, and we get the mode shape shown in Fig. 7a. For the anti-symmetric mode, the phase shift of the short cantilever is $-90^{\circ}$ while that of the long cantilever is $-270^{\circ}$. The difference of $180^{\circ}$ between the phase of the two cantilevers means that their vibrations are out-of-phase, and we get the mode shape shown in Fig. 7b.

\section{Mass detection}

In order to highlight the phenomenon of mode localization used for mass detection, a small mass perturbation of $\tilde{m}=6 \mathrm{pg}$ and representing $0.1 \%$ of the cantilever mass is added at the end of the long cantilever $\left(\tilde{x}_{m}=100 \mu m\right)$. After that, the theoretical amplitude 

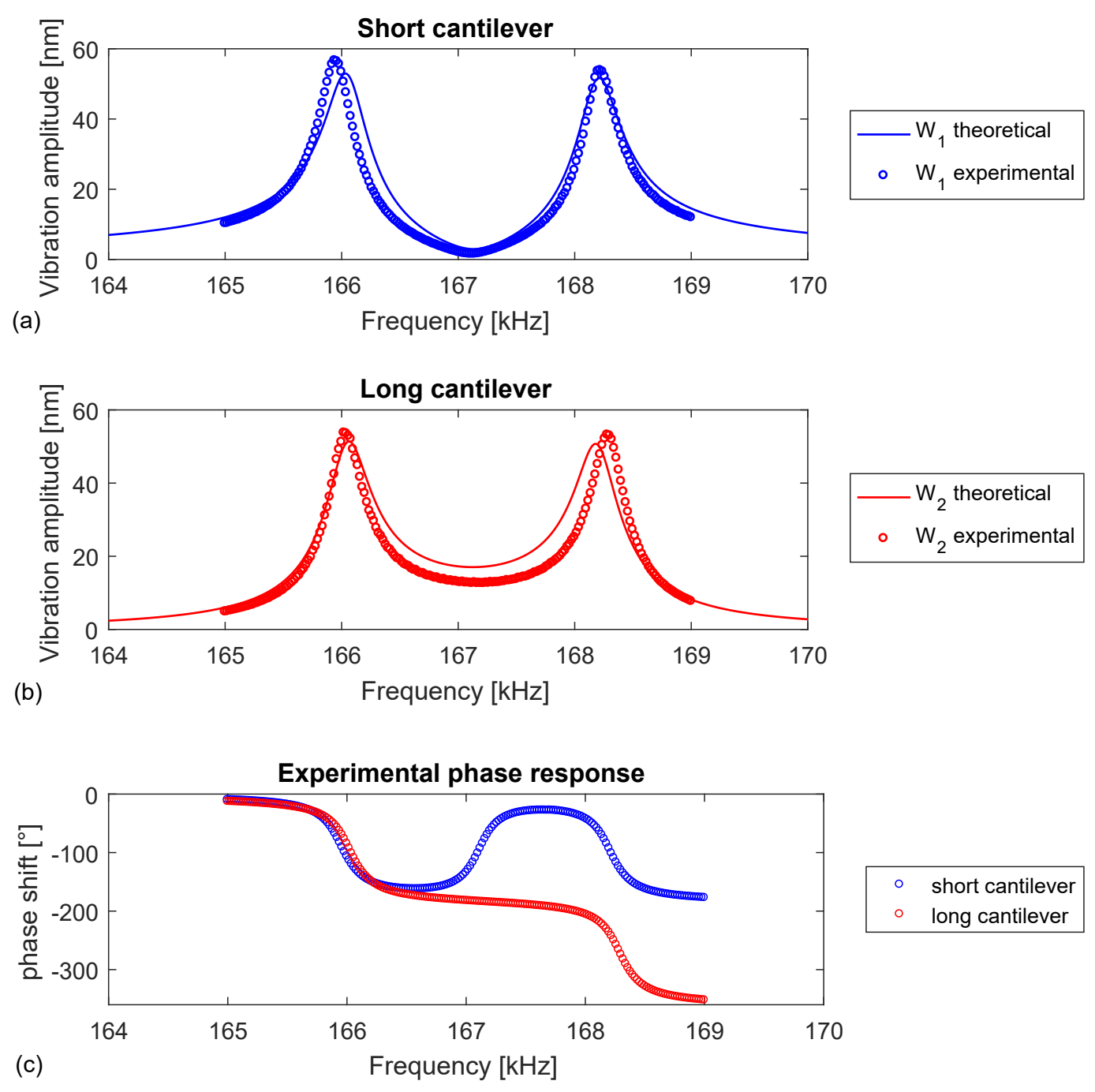

Figure 8: Frequency responses of the device 2 for $v_{A C=10 \mathrm{mV}}$ and $V_{D C}=5.85 \mathrm{~V}$ : amplitude response of the short cantilever $\left(L_{1}=98 \mu \mathrm{m}\right)(\mathrm{a})$, amplitude response of the long cantilever $\left(L_{2}=100 \mu \mathrm{m}\right)(\mathrm{b})$ and experimental phase response of the two cantilevers (c).

response is plotted, as shown in Fig. 9.

At the veering point, we remind that we have identical amplitude on both cantilevers. After adding the mass perturbation, we notice in Fig. 9 that the vibrations become localized. The theoretical result with a coupling beam at $5.5 \mu \mathrm{m}$ shows the most localized vibrations because it corresponds to the weakest coupling. In order to investigate the sensitivity of the device, we consider this coupling and simultions are performed with a mass perturbations between $0 \mathrm{pg}$ and $30 \mathrm{pg}$. It represents a relative mass between $0 \%$ and $0.5 \%$. Eigenstate shift is often used as output metrics for mode localized sensors [42] so we determine it for each added mass. It consists in calculating $\left|u_{i}-u_{0 i}\right| /\left|u_{0 i}\right|$, where $u_{0 i}$ and $u_{i}$ are respectively the eigenstate of the mode $i$ before and after adding the mass perturbation. The frequency variation of each mode is also calculated and results are shown in Fig. 10.

We note in Fig. 10a that the eigenstate shift is more important for the second mode and the sensitivity of the linear region is around $2.50 \% / \mathrm{pg}$. For the frequency variation, Fig. 10b 

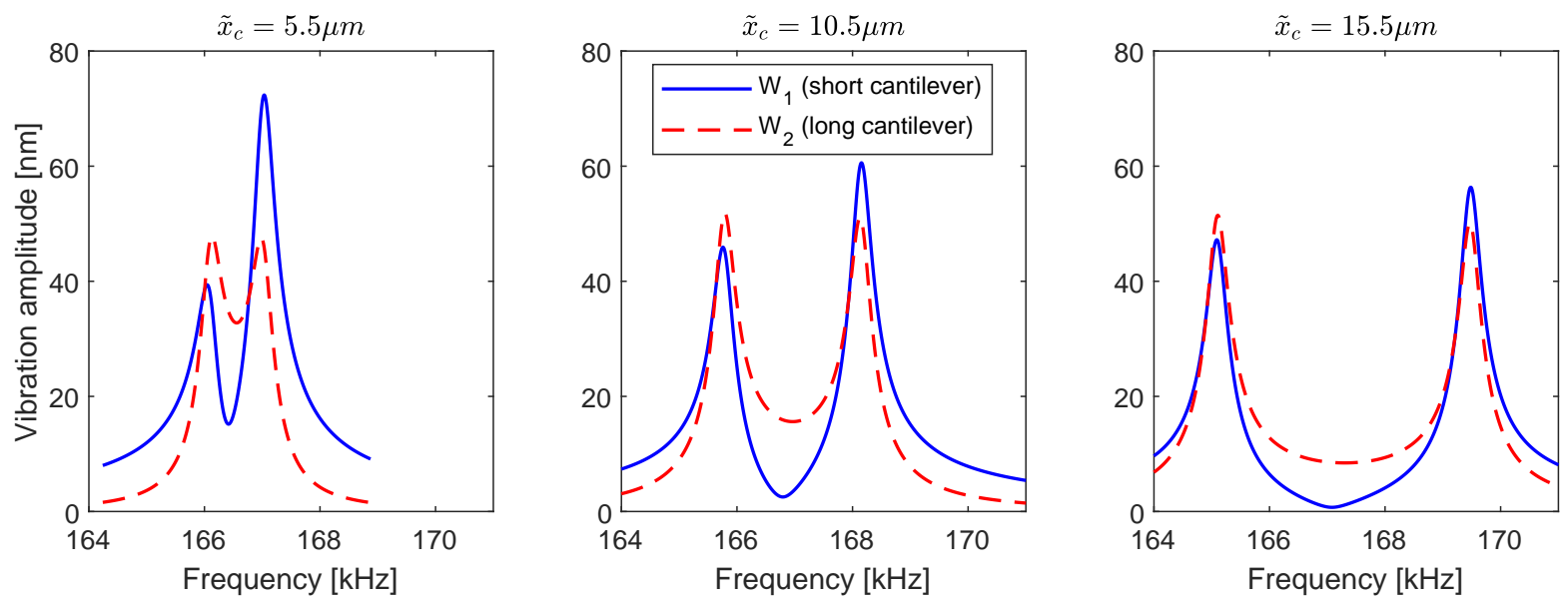

Figure 9: Theoretical amplitude responses with a mass perturbation of $6 \mathrm{pg}$ on the long cantilever

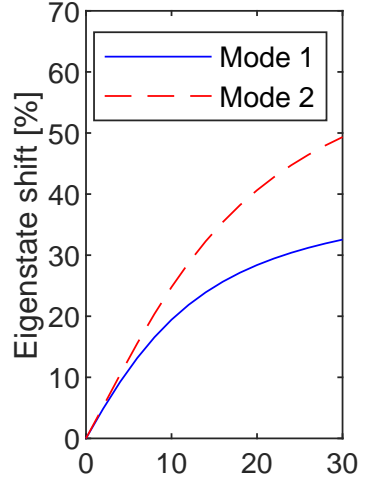

(a) Added mass [pg]

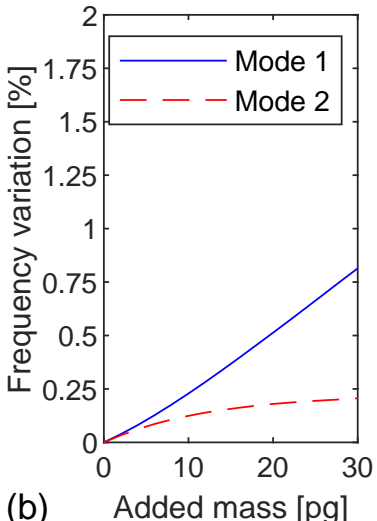

(b)

Figure 10: Eigenstate shift (a) and frequency variation (b) for $\tilde{x}_{c}=5.5 \mu \mathrm{m}$

shows that we have more variation in the first mode and the sensitivity is just around $0.03 \% / p g$.

\section{Conclusion}

A MEMS mass sensor composed of two cantilevers with different lengths and connected by a coupling beam is presented in this paper. The proposed model uses two Euler-Bernoulli beams coupled by a torsional spring and it is used to design the device. Experimental investigation is then carried out. By using the required DC voltage in each device, we have obtained the symmetric and the anti-symmetric modes at the veering point. These results show that it is possible to overcome the manufacturing defects by using a non-symmetrical system which can be then balanced by the electrostatic forces. The DC balancing voltage that we use is not the same for the three devices due to some disparities in the geometry, but it is close to the half of the pull-in voltage. Therefore, the length difference between the two cantilevers is appropriate for the device. Some parameters of the model are identified 
experimentally at the veering point, but using these same parameters with other actuation voltages leads to results close to those of the experiments. By changing the coupling between the two cantilevers, we still have good agreement between experimental and theoretical results. This confirms that the modelization of the mechanical coupling is efficient. Therefore, it can be used to find the optimal coupling which will give the highest sensitivity for the sensor. Finally, the proposed model is used to investigate the mode localization after adding a mass perturbation. The eigenstate shift gives a sensitivity which is two orders of magnitude higher than the frequency variation sensitivity. Future works will consist in the experimental characterization of the sensor under mass perturbations.

\section{Acknowledgments}

This project has been performed in cooperation with the EUR EIPHI program (ANR 17-EURE-0002)

\section{References}

[1] B. Ilic, D. Czaplewski, M. Zalalutdinov, H. Craighead, P. Neuzil, C. Campagnolo, C. Batt, Single cell detection with micromechanical oscillators, Journal of Vacuum Science \& Technology B: Microelectronics and Nanometer Structures Processing, Measurement, and Phenomena 19 (6) (2001) 2825-2828.

[2] A. Gupta, D. Akin, R. Bashir, Single virus particle mass detection using microresonators with nanoscale thickness, Applied Physics Letters 84 (11) (2004) 1976-1978.

[3] L.-T. Chen, C.-Y. Lee, W.-H. Cheng, Mems-based humidity sensor with integrated temperature compensation mechanism, Sensors and Actuators A: Physical 147 (2) (2008) $522-528$.

[4] A. Hajjam, J. Pandiyan, A. Rahafrooz, S. Pourkamali, Mems resonant sensors for detection of gasoline vapor, in: Sensors, 2010 IEEE, IEEE, 2010, pp. 1538-1541.

[5] J. Chaste, A. Eichler, J. Moser, G. Ceballos, R. Rurali, A. Bachtold, A nanomechanical mass sensor with yoctogram resolution, Nature nanotechnology 7 (5) (2012) 301.

[6] K. Ekinci, Y. Yang, M. Roukes, Ultimate limits to inertial mass sensing based upon nanoelectromechanical systems, Journal of applied physics 95 (5) (2004) 2682-2689.

[7] I. Kozinsky, H. C. Postma, I. Bargatin, M. Roukes, Tuning nonlinearity, dynamic range, and frequency of nanomechanical resonators, Applied Physics Letters 88 (25) (2006) 253101.

[8] N. Kacem, S. Hentz, D. Pinto, B. Reig, V. Nguyen, Nonlinear dynamics of nanomechanical beam resonators: improving the performance of nems-based sensors, Nanotechnology 20 (27) (2009) 275501.

[9] N. Kacem, J. Arcamone, F. Perez-Murano, S. Hentz, Dynamic range enhancement of nonlinear nanomechanical resonant cantilevers for highly sensitive nems gas/mass sensor applications, Journal of Micromechanics and Microengineering 20 (4) (2010) 045023. 
[10] W. Zhang, K. L. Turner, A mass sensor based on parametric resonance, in: Proceedings of the Solid State Sensor, Actuator and Microsystem Workshop, Hilton Head Island, SC, 2004, pp. 49-52.

[11] V. Kumar, J. W. Boley, Y. Yang, H. Ekowaluyo, J. K. Miller, G. T.-C. Chiu, J. F. Rhoads, Bifurcation-based mass sensing using piezoelectrically-actuated microcantilevers, Applied Physics Letters 98 (15) (2011) 153510.

[12] N. Kacem, S. Baguet, L. Duraffourg, G. Jourdan, R. Dufour, S. Hentz, Overcoming limitations of nanomechanical resonators with simultaneous resonances, Applied Physics Letters 107 (7) (2015) 073105.

[13] C. Zhao, M. H. Montaseri, G. S. Wood, S. H. Pu, A. A. Seshia, M. Kraft, A review on coupled mems resonators for sensing applications utilizing mode localization, Sensors and Actuators A: Physical 249 (2016) 93-111.

[14] P. W. Anderson, Absence of diffusion in certain random lattices, Physical review 109 (5) (1958) 1492.

[15] O. O. Bendiksen, Mode localization phenomena in large space structures, AIAA journal 25 (9) (1987) 1241-1248.

[16] P. Thiruvenkatanathan, J. Yan, A. A. Seshia, Common mode rejection in electrically coupled mems resonators utilizing mode localization for sensor applications, in: Frequency Control Symposium, 2009 Joint with the 22nd European Frequency and Time Forum. IEEE International, IEEE, 2009, pp. 358-363.

[17] M. Spletzer, A. Raman, A. Q. Wu, X. Xu, R. Reifenberger, Ultrasensitive mass sensing using mode localization in coupled microcantilevers, Applied Physics Letters 88 (25) (2006) 254102.

[18] P. Thiruvenkatanathan, J. Yan, J. Woodhouse, A. Aziz, A. Seshia, Ultrasensitive modelocalized mass sensor with electrically tunable parametric sensitivity, Applied Physics Letters 96 (8) (2010) 081913.

[19] T. Chopard, V. Lacour, T. Leblois, Gaas coupled micro resonators with enhanced sensitive mass detection, Sensors 14 (12) (2014) 22785-22797.

[20] Y. Wang, C. Zhao, C. Wang, D. Cerica, M. Baijot, Q. Xiao, S. Stoukatch, M. Kraft, A mass sensor based on 3-dof mode localized coupled resonator under atmospheric pressure, Sensors and Actuators A: Physical 279 (2018) 254-262.

[21] M. Spletzer, A. Raman, H. Sumali, J. P. Sullivan, Highly sensitive mass detection and identification using vibration localization in coupled microcantilever arrays, Applied Physics Letters 92 (11) (2008) 114102.

[22] C. Zhao, A mems sensor for stiffness change sensing applications based on three weakly coupled resonators, Ph.D. thesis, University of Southampton (2016). 
[23] H. Zhang, J. Yang, W. Yuan, H. Chang, Linear sensing for mode-localized sensors, Sensors and Actuators A: Physical 277 (2018) 35-42.

[24] H. Zhang, B. Li, W. Yuan, M. Kraft, H. Chang, An acceleration sensing method based on the mode localization of weakly coupled resonators, Journal of microelectromechanical systems 25 (2) (2016) 286-296.

[25] V. Walter, G. Bourbon, P. Le Moal, N. Kacem, J. Lardiès, Electrostatic actuation to counterbalance the manufacturing defects in a mems mass detection sensor using mode localization, Procedia Engineering 168 (2016) 1488-1491.

[26] T. Rabenimanana, V. Walter, N. Kacem, P. Le Moal, J. Lardiès, Nonlinear analytical model of two weakly coupled mems cantilevers for mass sensing using electrostatic actuation, in: Multidisciplinary Digital Publishing Institute Proceedings, Vol. 2, 2018, p. 1084.

[27] S. Timoshenko, Strength of materials Part 1, D. Van Nostrand Co., Inc, 1940.

[28] B. K. Hammad, Natural frequencies and mode shapes of mechanically coupled microbeam resonators with an application to micromechanical filters, Shock and Vibration 2014.

[29] G. Rinaldi, M. Packirisamy, I. Stiharu, Quantitative boundary support characterization for cantilever mems, Sensors 7 (10) (2007) 2062-2079.

[30] Z.-Y. Zhong, W.-M. Zhang, G. Meng, Dynamic characteristics of micro-beams considering the effect of flexible supports, Sensors 13 (12) (2013) 15880-15897.

[31] L. Bergman, D. M. McFarland, On the vibration of a point-supported linear distributed system, Journal of vibration, acoustics, stress, and reliability in design 110 (4) (1988) 485-492.

[32] S. INCEO Ǧ, M. Gürgöze, Bending vibrations of beams coupled by several double springmass systems, Journal of Sound and Vibration 243 (2) (2001) 370-379.

[33] L. Majkut, Eigenvalue based inverse model of beam for structural modification and diagnostics: theoretical formulation, Latin American Journal of Solids and Structures 7 (4) (2010) 423-436.

[34] H. Nishiyama, M. Nakamura, Form and capacitance of parallel-plate capacitors, IEEE Transactions on Components Packaging and Manufacturing Technology Part A 17 (3) (1994) 477-484.

[35] A. Bouchaala, A. H. Nayfeh, M. I. Younis, Frequency shifts of micro and nano cantilever beam resonators due to added masses, Journal of Dynamic Systems, Measurement, and Control 138 (9) (2016) 091002.

[36] G. O’Brien, D. J. Monk, L. Lin, Mems cantilever beam electrostatic pull-in model, in: Design, Characterization, and Packaging for MEMS and Microelectronics II, Vol. 4593, International Society for Optics and Photonics, 2001, pp. 31-42. 
[37] K. Naeli, O. Brand, Dimensional considerations in achieving large quality factors for resonant silicon cantilevers in air, Journal of applied Physics 105 (1) (2009) 014908.

[38] W. Zhang, K. L. Turner, Pressure-dependent damping characteristics of microsilicon beam resonators for different resonant modes, in: SENSORS, 2005 IEEE, IEEE, 2005, pp. $4-$ pp.

[39] Z. Hao, A. Erbil, F. Ayazi, An analytical model for support loss in micromachined beam resonators with in-plane flexural vibrations, Sensors and Actuators A: Physical 109 (1-2) (2003) 156-164.

[40] C. Pierre, Mode localization and eigenvalue loci veering phenomena in disordered structures, Journal of Sound and Vibration 126 (3) (1988) 485-502.

[41] B. K. Hammad, E. M. Abdel-Rahman, A. H. Nayfeh, Modeling and analysis of electrostatic mems filters, Nonlinear Dynamics 60 (3) (2010) 385-401.

[42] C. Zhao, G. Wood, J. Xie, H. Chang, S.-H. Pu, M. Kraft, Comparative study of different output metrics for a three weakly coupled resonator sensor, in: 2015 Transducers2015 18th International Conference on Solid-State Sensors, Actuators and Microsystems (TRANSDUCERS), IEEE, 2015, pp. 2196-2199. 